\title{
The precision calibration of depth and resolution for multibeam sonar in anechoic pool in the laboratory
}

\author{
Bei Shi ${ }^{1,2}$, Yongwei Liu ${ }^{1,2,3,}$, Dejiang Shang ${ }^{1,2,3}$, Xianwen Zhao ${ }^{1,2,3}$, and Yalin $\mathrm{Li}^{1,2,3}$ \\ ${ }^{1}$ Acoustic Science and Technology Laboratory, Harbin Engineering University, Harbin 150001, China \\ ${ }^{2}$ Key Laboratory of Marine Information Acquisition and Security (Harbin Engineering University), Ministry of Industry and \\ Information Technology; Harbin 150001, China \\ ${ }^{3}$ College of Underwater Acoustic Engineering, Harbin Engineering University, Harbin 150001, China
}

\begin{abstract}
The multibeam sonar is an important device for underwater survey, of which the measurement precision is a key parameter. We had designed an equipment to calibrate the depth and resolution precision for multibeam sonar in the anechoic pool in the laboratory. The main frame of the equipment is built up by circular and square steel pipes. The absorption materials are laid outside of the pipes, so that the multibeam sonar cannot receive the strong enough reflected acoustic waves from the boundary. We had made a two-dimensional scanning mechanism, which ascends and descends freely for evaluating the depth precision of multibeam sonar. The minimum precision of the depth is $0.1 \mathrm{~mm}$. We had set up a series of iron blocks with the dimension of $0.1,0.2,0.4,0.6,0.8$, and 1.0 square meters, respectively, as the standard objects for evaluating the resolution capability of multibeam sonar. In addition, we had placed an underwater camera system to record the iron blocks in time. The result of iron blocks measured by the calibrated multibeam sonar is compared to that recorded by the camera. Because the wavelength of acoustics is larger than that of optics, therefore, good precision of the resolution can be gotten.
\end{abstract}

\section{Introduction}

The multibeam sonar is a kind of high-tech products, which has been widely used in the survey of seabed topography and the depth of the sea[1]. Compared to the traditional sonar, the multibeam sonar has the advantages of high precision, wide measurement range and fast measurement speed[2]. And new algorithms make multibeam sonar get high resolution[3,4,5]. In the middle of 1960s, the concept of multibeam sonar was first proposed at the academic conference, which was held by American Woods Hole Institute of Oceanography. The world's first practically used multibeam sonar is the SeaBeam system, which was produced by General Electric Company in America. The SeaBat7122[6] multibeam sonar developed by Danish Reson company can generate up to 256 equal spacing beams. In the middle of 1990 s, the first multibeam sonar in China was developed by many scientific research institutes, which are leaded by Harbin Engineering University. In 2006, Harbin Engineering University developed the first portable highresolution shallow-water multibeam sonar[3] with the maximum detection depth of $200 \mathrm{~m}$. The technology of multibeam sonar has been rapidly developed and highly integrated by many kinds of high and new techniques, such as computer, navigation, location, and digital sensors.

The International Hydrographic Organization Standards for Hydrographic Surveys (IHO S-44 in short) was developed in 1994 and is internationally recognized as the standard of hydro-graphic survey. In IHOS-44, the minimum standard of hydro-graphic survey and the quantized index for measurement error are provided. The highest level is that the detection system must identify the target, of which the volume is bigger than 1 cubic meter and placed in the water with the depth of 40 meters. The IHO S-44 gives error parameters and confidence interval for the precision of depth and horizontal position. However, these criteria cannot meet the requirement for the calibration of the precision of the depth and resolution.

At present, the calibration experiments of multibeam sonar are mainly carried out in free-field waters. The accuracy of the measurement is affected by the factors, such as the errors of the installation, the fluctuation of water surface, the change of the temperature and the carrier's motion. There is no reliable calibration system for multibeam sonar in anechoic pool in the laboratory.

Based on the anechoic pool, we had proposed the idea that the calibration of the depth and resolution of multibeam sonar is carried out by the optical image, of which the accuracy and resolution is high.

In this paper, we had introduced the structure design of the calibration system for multibeam sonar. We had designed the main frame and the optimization of identification targets by the software. Through the numerical simulation by the ANSYS, we had completed the design of low reflection material. To meet the requirements for precision calibration of the depth and resolution, we had designed the scanning device. The results in the paper are useful to build the calibration system for multibeam sonar in the laboratory.

\footnotetext{
* Corresponding author: liuyongwei3000@hrbeu.edu.cn
} 


\section{Theoretical background}

\subsection{The work principle of multibeam sonar}
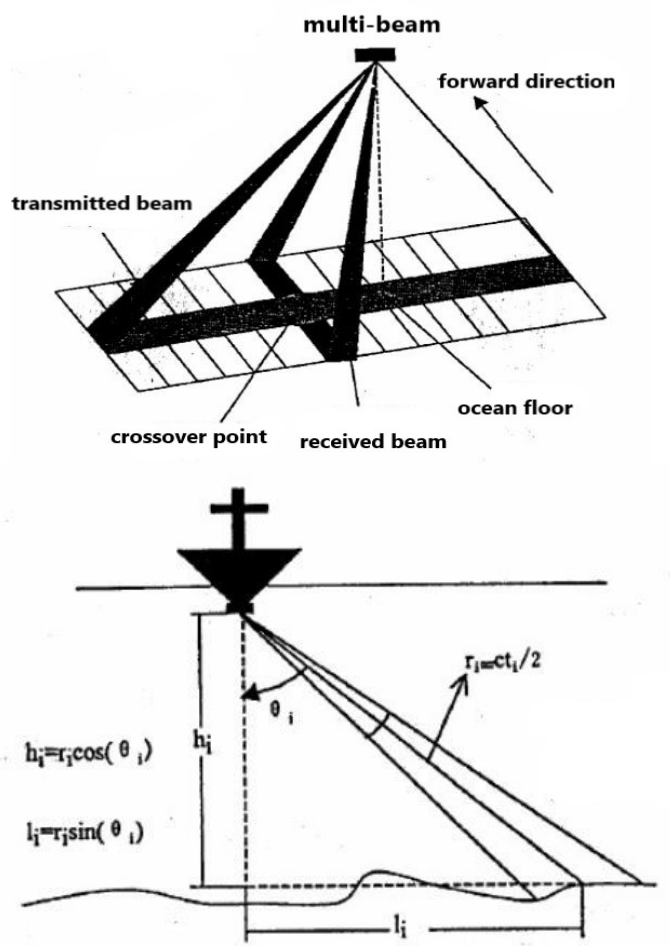

Fig. 1. The work principle of multibeam sonar.

The multibeam sonar transmits and receives acoustic signals through the vertical transmitting array and receiving array[1,7]. The transmitting array launches sector-shaped beams with wide-transverse and narrow longitudinal power along the motion direction of multibeam sonar, the large coverage of underwater detection can be achieved. At the same time, the receiving array receives the narrow-transverse and widelongitudinally beams along the motion direction of multibeam sonar. The transmitted beams and the received beams of multibeam sonar form the crossover point, which can be measured. When the multibeam sonar is moving, the locations of many crossover points will be gotten.

$$
\begin{aligned}
& h=\frac{c t}{2} \cos (\theta) \\
& l=\frac{c t}{2} \sin (\theta)
\end{aligned}
$$

where, $h$ is the depth of the measured point, $l$ is the horizontal relative position of the measured point. We can stitch the measurement results and get the topographic map of the area, when the multibeam sonar is working along the specified measurement line.

\subsection{The evaluation of sounding data}

As the table shows, the IHO S-44 gives the lowest standard of the measurement for different levels. To calculate the accuracy of the detection, we can use the limit error and formula in the table. But in practical applications, the evaluation of the measurement results by the absolute depth of the water and some other relative factors is not reliable. Therefore, in the practical calibration process, it is necessary to find a suitable measurement method to evaluate the results when we have gotten the measured data.

Table 1. Minimum Standards for Hydro-graphic Surveys.

\begin{tabular}{|c|c|c|c|c|}
\hline Order & Special & $\mathbf{1 a}$ & $\mathbf{1 b}$ & $\mathbf{2}$ \\
\hline $\begin{array}{c}\text { Maximum allowable } \\
\text { THU } \\
\mathbf{9 5 \%} \text { Confidence level) }\end{array}$ & 2 meters & $\begin{array}{c}5 \text { meters }+5 \% \text { of } \\
\text { depth }\end{array}$ & $\begin{array}{c}5 \text { meters }+ \\
5 \% \text { of depth }\end{array}$ & $\begin{array}{c}20 \text { meters }+ \\
10 \% \text { of depth }\end{array}$ \\
\hline $\begin{array}{c}\text { Maximum allowable } \\
\text { TVU }\end{array}$ & $\begin{array}{c}\mathrm{a}=0.25 \text { meter } \\
\mathbf{b}=0.0075\end{array}$ & $\begin{array}{c}\mathrm{a}=0.5 \text { meter } \\
\mathrm{b}=0.013\end{array}$ & $\begin{array}{c}\mathrm{a}=0.5 \text { meter } \\
\mathrm{b}=0.013\end{array}$ & $\begin{array}{c}\mathrm{a}=1.0 \text { meter } \\
\mathrm{b}=0.023\end{array}$ \\
\hline Feature Detection & $\begin{array}{c}\text { Cubic features }> \\
1 \text { meter }\end{array}$ & $\begin{array}{c}\text { Cubic features }>2 \\
\text { meters, in depths up to } \\
40 \text { meters; } 10 \% \\
\text { Of depth beyond } 40 \\
\text { meters }\end{array}$ & $\begin{array}{c}\text { Not } \\
\text { Applicable }\end{array}$ & $\begin{array}{c}\text { Not } \\
\text { Applicable }\end{array}$ \\
\hline
\end{tabular}

The static measurement is that we measure the same position several times and calculate the data of the depth with the multibeam sonar in the static state. We can evaluate the detection precision by the calculation of one position's mean value and standard deviation of the data with the method of statistics.

The depth of the $i$ th time measurement is $h_{i}$, and $N$ is the total number of measurement times, so the mean value of the depth is

$$
\bar{h}=\frac{\sum_{i=1}^{N} h_{i}}{N}
$$

The standard deviation of the depth is

$$
\sigma=\sqrt{\sum_{i=1}^{N}\left(h_{i}-\bar{h}\right)^{2} / N}
$$

\section{The design of multibeam sonar calibration system}

\subsection{The general design of the system}


As shown in the Fig.2, the multibeam sonar calibration system includes three parts: the main frame of the equipment, which is built up by circular and square steel pipes, the two-dimensional precision scanning mechanical system and a series of iron blocks. According to the size of the anechoic pool, we design the frame of the equipment with the length of $4.3 \mathrm{~m}$, width of $4.1 \mathrm{~m}$ and the height of $5.6 \mathrm{~m}$. In order to transport conveniently, the frame consists with several parts. The length of the longest part is $5.2 \mathrm{~m}$ and the total mass of the frame is $2600 \mathrm{~kg}$.

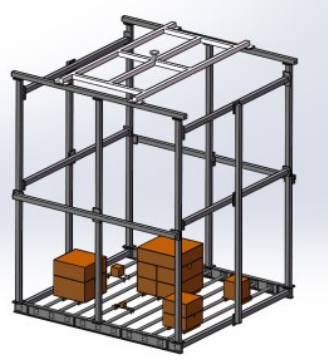

Fig. 2. The calibration system of multibeam sonar

There is a temperature measurement device in the anechoic pool, so that we can calculate the sound velocity by the temperature and modify the parameter of multibeam sonar.
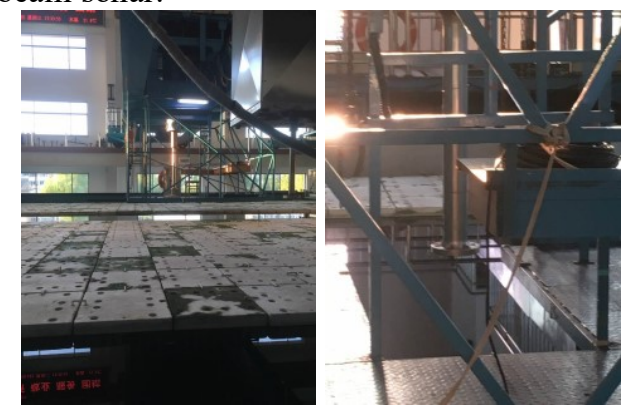

Fig. 3. The anechoic pool.

\subsection{The design of low reflection material}

The frame of the equipment is built up by square steel pipes. Several circular pipes are equally placed at the bottom of the frame. The absorption materials are laid outside of the pipes.

The design of the anechoic coatings is mainly focused in the low frequency range. However, in high frequency range, we only have the common sense that because of viscosity-elastic deformation of the materials, sound energy will be converted into heat, thus, the materials can reduce the reflected sound waves.

Based on the Block's periodical theory, we had created the model of wave-shaped anechoic coatings and calculated the simulation by the software, ANSYS. The material of the anechoic coatings is silica gel. The coefficient of sound absorption and sound reflection in the sound field are averaged, then, we had gotten that the coefficient of sound absorption of wave-shaped coatings is equal to 0.982 at the frequency of $100 \mathrm{kHz}$. It shows that the wave-shaped coatings laid on the frame are feasible to reduce the reflected sound waves. Therefore, the problem of the reflection effect of the frame is solved.
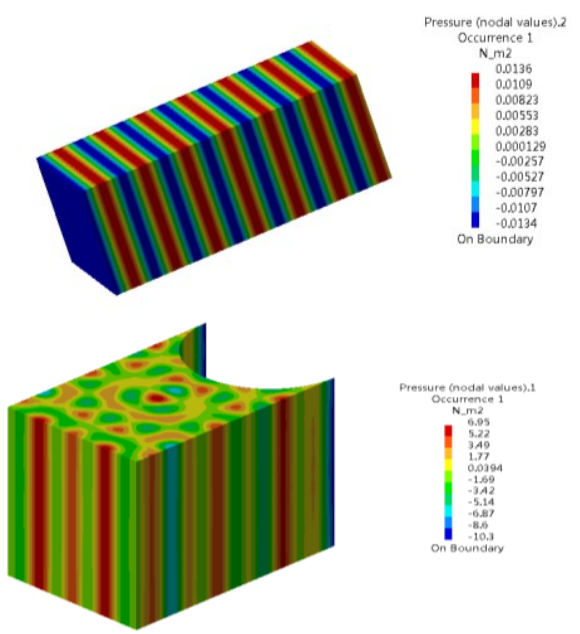

Fig. 4. The diagram of the anechoic coatings' calculation

\subsection{The accuracy design of the depth}

The IHOS-44 gives the formula to calculate the maximum allowable Total vertical uncertainty (TVU) for a specific depth

$$
\pm \sqrt{\left.\left[a^{2}+(b \times d)^{2}\right)\right]}
$$

where, $a$ is the portion of the uncertainty that does not vary with the depth, $b$ is the coefficient which represents the portion of the uncertainty that varies with depth, $d$ is the depth.

To calibrate the multibeam sonar, a series of iron blocks are set up at the bottom of the frame, these blocks are no weight in the water because they are hollow. We had built up the static models of these blocks in the software, ANSYS. According to the result of simulation, we get the deformation of the circular pipes when the blocks were set up.

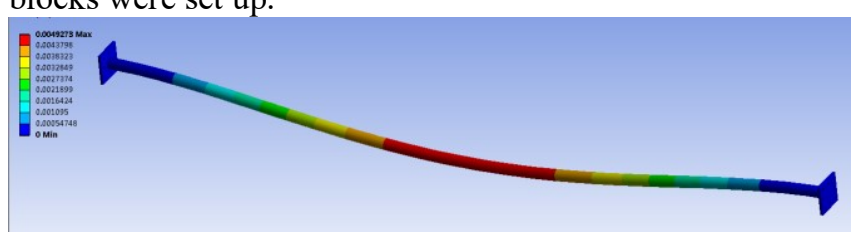

Fig. 5. The change of the force induced by the iron block on the circular pipe.

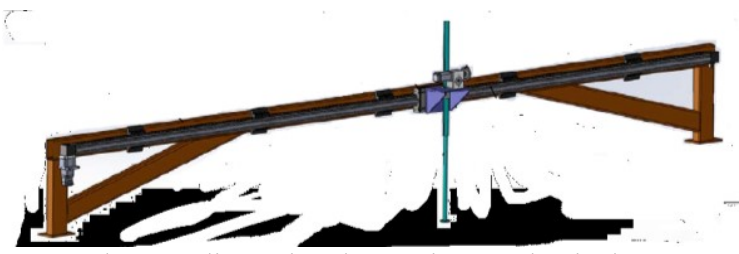

Fig. 6. The two-dimensional scanning mechanical structure.

The maximum load on the single circular pipe is $1300 \mathrm{~N}$. Because the load is uneven, we had calculated the deformation of the pipe under the load of $2000 \mathrm{~N}$. The amplitude of the deformation is so little and in elastic range that the pipe will be recovered. When the blocks are put in the water, the buoyancy of the blocks 
will balance its gravity and the pipes will have no load and not be deformed.

To achieve the precision calibration of the system, we must control the multibeam sonar precisely in the vertical displacement. A two-dimensional scanning mechanical structure consists of the track, electronic control system, and two stepping motors. The stepping motors control the horizontal movement and vertical movement of the device. The multibeam sonar scans and identifies the targets in the horizontal movement, and we can adjust the vertical position of the device and target in the vertical movement. We can get the vertical relative displacement between the top of the pole and the top of the target by the laser rangefinder, the distance is $d_{1}$. Then, the stepping motor moves to another vertical distance, $d_{2}$. Therefore, the distance of the relative movement is

$$
D=d_{2}-d_{1}
$$

The accuracy of the laser rangefinder can be $0.1 \mathrm{~mm}$, and the accuracy of vertical displacement control is also $0.1 \mathrm{~mm}$, so that the measurement accuracy of the vertical distance between the top of the pole and the top of the target is also $0.1 \mathrm{~mm}$. The advantage of the relative distance calculated by the formula to calibrate the multibeam sonar is that we can eliminate the errors caused by the deformation when the frame structure is placed in the water.

\subsection{The design of the targets}

Based on the criteria of the IHOS-44 and JT/T 790-2010, at the bottom of the frame of the multibeam sonar calibration system, we set up 6 iron blocks with the dimension of $0.1,0.2,0.4,0.6,0.8$ and 1.0 square meters as the standard objects. Considered the limited load capacity of the frame, if the stainless-steel target is solid, the weight is too large. Therefore, each dimension target is optimally designed.

Each target has an exhaust holes, water inlets, hollow layer and installation device. The targets will be suspended when they are in the water.
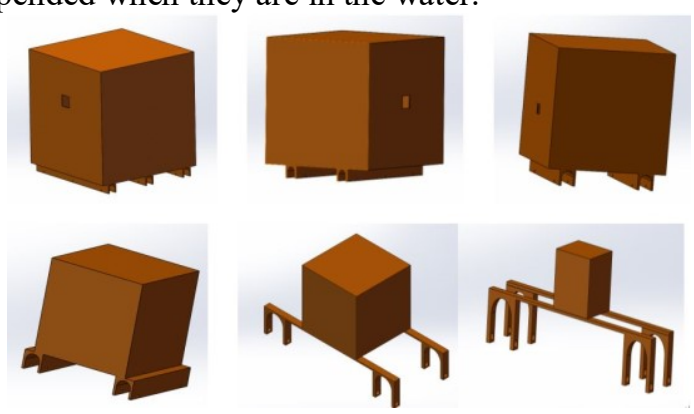

Fig. 7. The targets with the dimension of $0.1,0.2,0.4,0.6,0.8$ and 1.0 square meter。

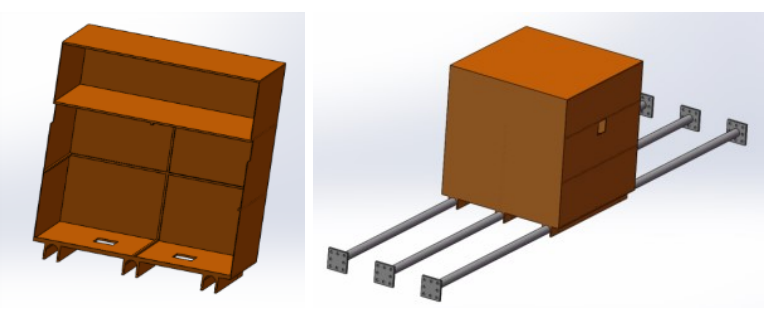

Fig. 8. The target $(1 \mathrm{~m})$.

The Fig. 8 shows that the length of the target is $1 \mathrm{~m}$. To reduce its weight, the thickness is $3 \mathrm{~mm}$. There are several stiffened ribs in the targets. The upper part of the target is a hollow layer, which can provide buoyancy in the water. The water will flow into the lower part of the target through the water inlets.

Table 2. The weight of each target.

\begin{tabular}{|c|c|c|c|c|c|c|}
\hline Target & $\mathbf{1 . 0 m}$ & $\mathbf{0 . 8 m}$ & $\mathbf{0 . 6 m}$ & $\mathbf{0 . 4 m}$ & $\mathbf{0 2 m}$ & $\mathbf{0 . 1 m}$ \\
\hline $\begin{array}{c}\text { Weight } \\
\text { in air }\end{array}$ & $373 \mathrm{~kg}$ & $248 \mathrm{~kg}$ & $144 \mathrm{~kg}$ & $64 \mathrm{~kg}$ & $18 \mathrm{~kg}$ & $10 \mathrm{~kg}$ \\
\hline $\begin{array}{c}\text { Weight } \\
\text { in water }\end{array}$ & $39 \mathrm{~kg}$ & $11 \mathrm{~kg}$ & $24 \mathrm{~kg}$ & $0 \mathrm{~kg}$ & $8 \mathrm{~kg}$ & $10 \mathrm{~kg}$ \\
\hline
\end{tabular}

\section{Conclusions}

We had built up the system to calibrate the precision of the depth and resolution for multibeam sonar in the anechoic pool in the laboratory. The calibration system sets up the targets at the bottom of the frame, and uses the relative movement of the device to calibrate the depth accuracy of multibeam sonar. The accuracy of the depth can be $0.1 \mathrm{~mm}$ by the application of the laser rangefinder. The different sizes of iron blocks are selected as the identification targets, and calibrate the resolution of multibeam sonar by the comparison with the optical imaging results. The wave-shaped anechoic coatings lay outside of the frame and with the anechoic wedges in the anechoic pool, the multibeam sonar can work in a completely free-field environment in the calibration process, and the background interference caused by the boundary reflection will be eliminated.

The research of this paper provides the laboratory calibration system for the multibeam sonar, and lays the foundation for the establishment of the measurement standard for the multibeam sonar in the future.

The work has been supported by the National Key Research Development Project, No.2016YFF0200906, Heilongjiang Province Foundation, No.GX17A015, and China Postdoctoral Science Foundation Funded Project, No.2017M611358. Elsewhere, the work has been supported by the major project from Acoustic Science and Technology Laboratory, No.9140C200104140C20003, and also been funded the steady support plan from Acoustic Science and Technology Laboratory (Grant No.SSJSWDZC2018005). Furthermore, the work has been funded by the project from Key Laboratory of Acoustic Stealth (Grant No.614220405011706), the Fundamental Research Funds for Central universities (Grant No.HEUCF180503), and also funded by the China Scholarship Council, No.201706685061. 


\section{References}

[1] Baowei C, Research and implementation of the technology for super-Wide coverage multibeam bathymetry. Harbin: Har-bin Engineering University, 1-12 (2012)

[2] Haisen. L, Study of multibeam swath bathymetry survey system. Harbin: Harbin Engineering University, 1-10 (1999)

[3] ANDERSON J T, Acoustic seabed classification of marine physi-cal and biological landscapes. Denmark: International Council for the Exploration of the Sea (ICES) (1017-6195)94113(2007)

[4] LLORT-PUJOL G, SINTES C, CHONAVEL T, et al. Ma-rine Technology Society Journal, 46(2) 9-31( 2012)

[5] Kun Y, Yongting W, Tiehu Z, et al. Technology and application of marine investigation. Wuhan: Wuhan University Press, 40-48(2009)

[6] LLORT-PUJOL G, SINTES C, LURTON X, J. Acoust. Soc. Am., 123(5) 3952-3952 (2008)

[7] Haisen. L, Bin Y, Tian Z, et al. Shallow Water High Resolution Multibeam Echo Sounder. // KOBE: MTS/IEEE OCEANS, 1051-1055(2008) 